\section{LIPOSSARCOMA DE LARINGE: RELATO DE CASO}

\author{
LARYNGEAL LIPOSARCOMA: A CASE REPORT
}

Alexandre Carvalho Abud ${ }^{1}$, Thais Barbosa de Paula ${ }^{1}$, João Marcos Arantes Soares², Lívio Bruno Santos Cunha², Ana Jacinta de Aquino Peixoto ${ }^{2}$

\begin{abstract}
RESUMO
O lipossarcoma de laringe é uma neoplasia extremamente rara, acomete principalmente o sexo masculino, principalmente na quinta década de vida. Existindo apenas cerca de 40 casos descritos na literatura, desses nenhum em língua portuguesa. O presente caso relata o diagnóstico em um paciente do sexo masculino, 57 anos, ex-tabagista, apresentando alteração de voz e obstrução de via área. Foi optado por ressecção cirúrgica completa com achados sugestivos de lipossarcoma bem diferenciado. Foi optado por manter seguimento, não tendo sido indicado quimioterapia e radioterapia adjuvantes.
\end{abstract}

Palavras-chave: Lipossarcoma; lipossarcoma-laringe; cirurgia-cabeça-pescoço; sarcomas

\begin{abstract}
Laryngeal liposarcoma is an extremely rare neoplasm that affects especially men in the fifth decade of life. There are only about 40 cases described in the literature, none of them in the Portuguese language. We report the case of a 57-year-old, former smoker man presenting with voice disorders and airway obstruction. We opted for complete surgical resection with findings suggestive of well-differentiated liposarcoma. We chose to keep following the patient, and no adjuvant chemotherapy and radiotherapy were indicated.
\end{abstract}

Keywords: Liposarcoma; laryngeal liposarcoma; head and neck surgery; sarcomas

Os lipossarcomas são tumores mesenquimais malignos originados de tecido adiposo, foram primeiramente descritos por Virchow em 1857 e posteriormente foram classificados por Enzinger e Weis em quatro subtipos histológicos ${ }^{1}$. Tal classificação compreende os seguintes subtipos: tumor lipomatoso bem diferenciado, mixoide, pleomórfico e desdiferenciado ${ }^{2,3}$. Desses subtipos, o bem diferenciado é o mais comum.

O lipossarcoma é o sarcoma mais comum da vida adulta, representando $20 \%$ dos diagnósticos de sarcoma, acometendo principalmente membros inferiores e retroperitônio ${ }^{3,4}$. Desses, apenas $5 \%$ são encontrados na região de cabeça e pescoço e apenas uma pequena parcela é encontrada na laringe ${ }^{5,6}$.

O lipossarcoma de laringe atinge principalmente o sexo masculino, acometendo qualquer faixa etária, principalmente na quinta década de vida com idade média de apresentação de 46 anos $^{7}$. Trata-se de uma neoplasia extremamente rara - existindo aproximadamente 40 casos descritos na literatura, desses nenhum em língua portuguesa ${ }^{2,4}$.

\section{RELATO DO CASO}

Paciente do sexo masculino, 57 anos, atendido no serviço de cirurgia de cabeça e pescoço do Complexo de Saúde de São João de Deus, em Divinópolis-MG, em janeiro de 2016, com relato de tumoração na região cervical com crescimento rápido nos últimos cinco meses. Negava dor, disfagia, dispneia e disfonia. Hipertenso, ex-tabagista e etilista moderado.
Clin Biomed Res. 2019;39(3):252-253

1 Faculdade de Medicina, Universidade Federal de São João del-Rei (UFSJ). São João del-Rei, MG, Brasil.

2 Departamento de Cirurgia, Hospital São João de Deus (HSJD). Divinópolis, MG, Brasil.

Autor correspondente: Alexandre Carvalho Abud alexandre.abud.ufsj@gmail.com Faculdade de Medicina, Universidade Federal de São João del-Rei (UFSJ) Praça Frei Orlando, 170. 36307-352, São João del-Rei, MG, Brasil. 
Videonasolaringoscopia evidenciou lesão submucosa à direita da parede lateral da orofaringe até as estruturas da supraglote.

Realizada PAAF com resultado insuficiente para definição diagnóstica. Optado então por realização de biópsia sob narcose com achados sugestivos de lesão mesenquimal gordurosa bem diferenciada (lipoma).

Paciente evoluiu com disfonia e posterior obstrução de via aérea, sendo submetido a traqueostomia de urgência em maio de 2016 e submetido a abordagem cirúrgica em julho do mesmo ano.

Realizada ressecção completa da lesão, que se insinuava pela membrana tireohioidea e abaulava extrinsecamente a faringe, no nível do seio piriforme e obstruía a via aérea ao nível da supraglote. A lesão apresentava planos anatômicos que a separavam das estruturas adjacentes, de modo que não foi necessária a ressecção de estruturas laríngeas. Permaneceu em uso de sonda nasoentérica por três semanas após o procedimento, quando foi reintroduzida a dieta oral habitual, sem relato de disfagia. Um mês após o tratamento cirúrgico paciente foi decanulado, sem intercorrências. Estudo anatomopatológico evidenciou achados compatíveis com lipossarcoma bem diferenciado, ressecado com margens livres.
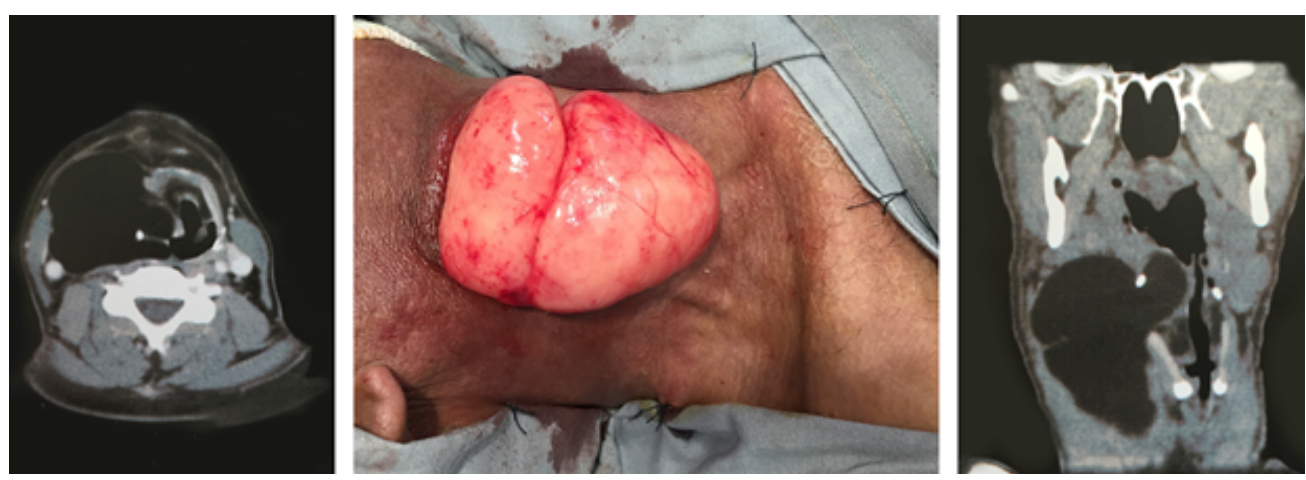

Figura 1: Paciente do sexo masculino com relato de tumoração na região cervical.
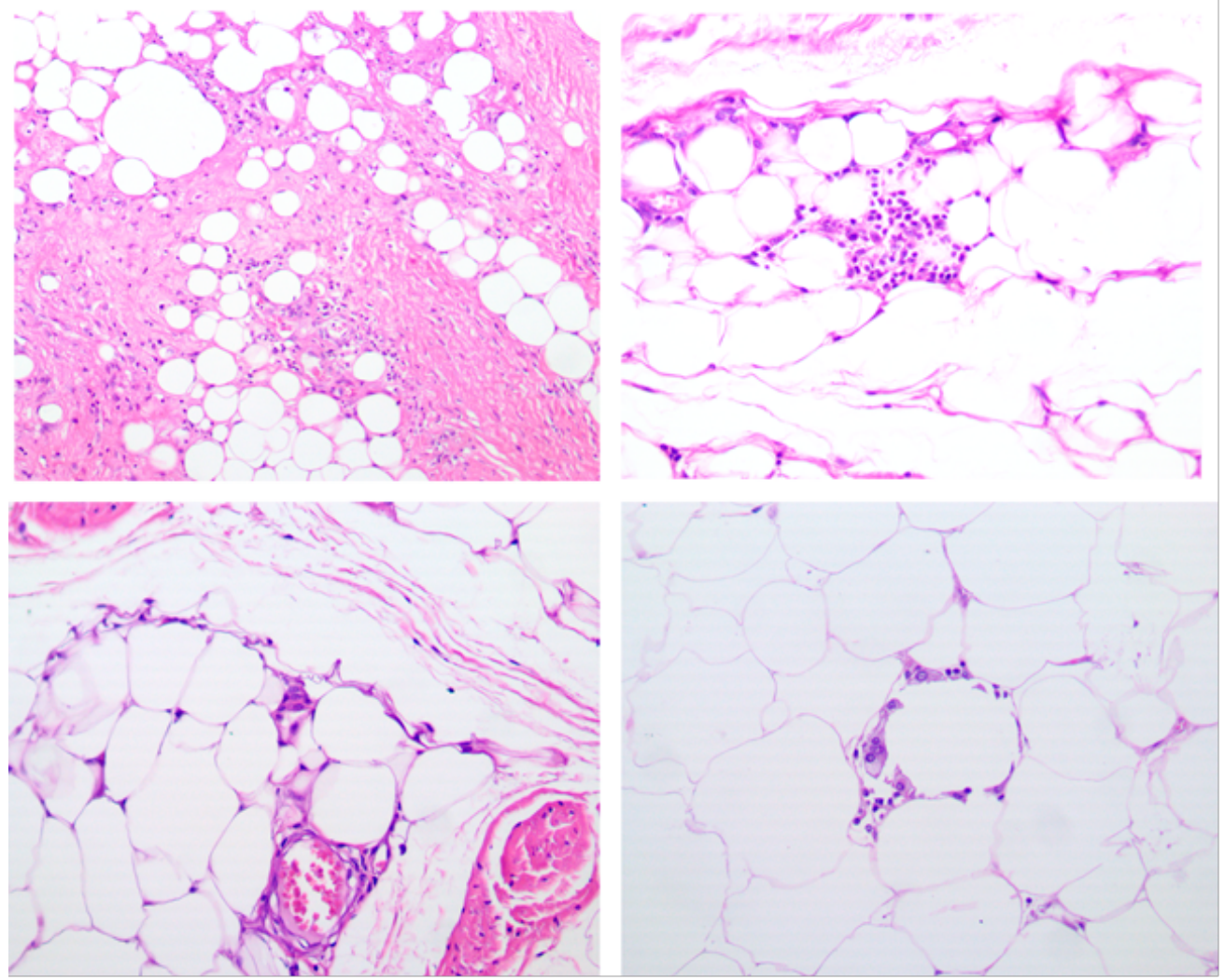

Figura 2: Estudo anatomopatológico. 
Optou-se por manter seguimento, não tendo sido indicado quimioterapia e radioterapia adjuvantes. Paciente segue há mais de três anos sem evidência de recorrência locorregional.

\section{DISCUSSÃO}

Cerca de $75 \%$ dos lipossarcomas de laringe ocorrem na região supraglótica. Geralmente têm formato polipoide e o tamanho máximo descrito na literatura é de $9 \mathrm{~cm}$ em seu maior diâmetro. Os sintomas mais comuns são disfagia, disfonia, odinofagia, ronco e dispneia devido à obstrução significativa das vias aéreas ${ }^{1,6,7}$.

O diagnóstico do lipossarcoma de laringe representa um desafio, devido aos sintomas vagos, achados inespecíficos em exames de imagem e a natureza rara da doença, além de exibir um crescimento muito lento que pode ser facilmente confundido como uma lesão benigna. Assim, o exame anatomopatológico é o padrão ouro e, mesmo na histologia, o tipo bem diferenciado pode ser confundido com um simples lipoma ${ }^{2,6}$.

O principal tratamento do lipossarcoma é a excisão cirúrgica, por meio de ressecção ampla e radical do tumor, visto que margens comprometidas estão associadas a um risco aumentado de recidiva. Porém, quando a lesão é extensa, a sua ressecção completa pode significar desfuncionalização da laringe, o que aumenta a morbidade do procedimento cirúrgico. O esvaziamento cervical eletivo não é comumente indicado, visto que a metástase nodal é extremamente rara ${ }^{2,5}$.

A laringectomia total deve ser indicada após múltiplas recorrências ou quando o tamanho e a localização do tumor impedem a obtenção de margens livres. Deve ser realizada quando não é possível preservar a função do órgão. Já o uso de tratamento adjuvante não é consenso na literatura ${ }^{4,5}$.

O prognóstico depende do grau histológico, do sítio do tumor e da profundidade da lesão. O tamanho não confere necessariamente pior prognóstico, mas está relacionado em maior dificuldade de ressecção completa. Os subtipos bem diferenciados e mixoide apresentam melhor prognóstico. De modo geral, o prognóstico dos lipossarcomas laríngeos parece ser consideravelmente mais favorável quando comparado a esse mesmo tumor em outras regiões. Este fato pode estar relacionado à baixa incidência de tumores de alto grau e a raridade de metástase ${ }^{1,7}$.

Estudos indicam que a associação de radioterapia ou quimioterapia ao tratamento cirúrgico não deve ser recomendado de rotina para pacientes com subtipos histológicos de bom prognóstico, uma vez que não melhora a sobrevida do paciente. A radioterapia deve ser reservada para pacientes com lesões de alto grau com grande extensão local, casos com alta recorrência e quando a ressecção completa não é possível ${ }^{1,2}$.

O emprego da quimioterapia ainda é incipiente, sendo a descarbazina e doxorrubicina drogas que apresentam melhores resultados no subtipo mixoide. Entretanto, não há efeito comprovado sob as formas bem diferenciada e desdiferenciada ${ }^{1}$.

O lipossarcoma de laringe é uma neoplasia extremamente rara e o diagnóstico correto é desafiador, sendo frequentemente confundido com lesões benignas e confirmado após recorrência local e revisão de exames anatomopatológicos já realizados. Desta forma, a publicação de novos casos deve ser encorajada a fim de proporcionar subsídio a trabalhos que levem ao aprimoramento do manejo dessa doença por meio do desenvolvimento de protocolos de tratamento e modalidades terapêuticas mais eficazes e seguras.

\section{REFERÊNCIAS}

1. Kodiyan J, Rudman JR, Rosow DE, Thomas GR. Lipoma and liposarcoma of the larynx: case reports and literature review. Am J Otolaryngol. 2015;36(4):611-5.

2. Han Y, Yang LH, Liu TT, Wang J, $\mathrm{Li} \mathrm{H}, \mathrm{Yu} \mathrm{G}$, et al. Liposarcoma of the larynx: report of a case and review of literature. Int J Clin Exp Pathol. 2014;8(1):1068-72.

3. Nassif NA, Tseng W, Borges C, Chen P, Eisenberg B. Recent advances in the management of liposarcoma. F1000Res. 2016;5:2907.
4. Zhu H, Sun J, Wei S, Wang D, Brandwein M. Well-differentiated laryngeal/hypopharyngeal liposarcoma in the MDM2 era report of three cases and literature review. Head Neck Pathol. 2017;11(2):146-51.

5. Gleinser DM, Font JP, Clement CG, Mohammed BS, Underbrink MP. Primary myxoid liposarcoma of the supraglottic larynx. Rare tumors. 2010;2(3):e41.

6. Farkas $A B$, House LK, Khan M, Saad AG, Parker E, Joyner D. Liposarcoma of the glottis: a report of an unusual diagnosis in an unusual location. J Radiol Case Rep. 2018;13(3):631-4.

7. Makeieff $M$, Pelliccia $P$, Poizat $F$, Arnaud S, Rat F, Cupissol D, et al. Laryngeal dedifferentiated liposarcoma. Eur Arch Otorhinolaryngol. 2010;267(6):991-4. 